\title{
Correction to: Evaluation of intraseasonal wind rectification on recent Indian Ocean dipole events using LICOM
}

\author{
Xia Zhao ${ }^{1,3,5} \cdot$ Dongliang Yuan ${ }^{1,2,3,4,5}$ (])
}

Published online: 15 February 2022

๑) Springer-Verlag GmbH Germany, part of Springer Nature 2022

\section{Correction to: Climate Dynamics https://doi.org/10.1007/s00382-021-05943-1}

In the original version of the article published online,

Xia Zhao ${ }^{1,2,3}$ and Dongliang Yuan ${ }^{1,2,3,4,5}$

${ }^{1}$ CAS Key Laboratory of Ocean Circulation and Waves, and Center for Ocean Mega- Science, Institute of Oceanology, Chinese Academy of Sciences, Qingdao, China.

${ }^{2}$ Key Laboratory of Marine Science and Numerical Modeling, First Institute of Oceanography, Ministry of Natural Resources, Qingdao, China.

${ }^{3}$ Pilot National Laboratory for Marine Science and Technology (Qingdao), Qingdao, China.

${ }^{4}$ Shandong Key Laboratory of Marine Science and Numerical Modeling, Qingdao, China.

Affiliation 1 and Affiliation 2 share the first position.

The original article can be found online at https://doi.org/10.1007/ s00382-021-05943-1.

Dongliang Yuan

dyuan@qdio.ac.cn

1 CAS Key Laboratory of Ocean Circulation and Waves, and Center for Ocean Mega- Science, Institute of Oceanology, Chinese Academy of Sciences, Qingdao, China

2 Key Laboratory of Marine Science and Numerical Modeling, First Institute of Oceanography, Ministry of Natural Resources, Qingdao, China

3 Pilot National Laboratory for Marine Science and Technology (Qingdao), Qingdao, China

4 Shandong Key Laboratory of Marine Science and Numerical Modeling, Qingdao, China

5 University of Chinese Academy of Sciences, Beijing, China
${ }^{5}$ University of Chinese Academy of Sciences, Beijing, China.

Author Zhao Xia should be indexed with Affiliation 5 instead of Affiliation 2. In addition, the statement "Affiliation 1 and Affiliation 2 share the first position" was missing. Please make the necessary correction to the online publication. They should look something like.

Xia Zhao ${ }^{1,3,5}$ and Dongliang Yuan ${ }^{1,2,3,4,5}$

${ }^{1}$ CAS Key Laboratory of Ocean Circulation and Waves, and Center for Ocean Mega- Science, Institute of Oceanology, Chinese Academy of Sciences, Qingdao, China.

${ }^{2}$ Key Laboratory of Marine Science and Numerical Modeling, First Institute of Oceanography, Ministry of Natural Resources, Qingdao, China.

${ }^{3}$ Pilot National Laboratory for Marine Science and Technology (Qingdao), Qingdao, China.

${ }^{4}$ Shandong Key Laboratory of Marine Science and Numerical Modeling, Qingdao, China.

${ }^{5}$ University of Chinese Academy of Sciences, Beijing, China.

Affiliation 1 and Affiliation 2 share the first position.

Publisher's Note Springer Nature remains neutral with regard to jurisdictional claims in published maps and institutional affiliations. 